\title{
EphA2 Promotes Infiltrative Invasion of Glioma Stem Cells in vivo through Crosstalk with Akt and Regulates Stem Properties
}

\author{
Hui Miao ${ }^{1,2,{ }^{*} \text {, Nickolas W. Gale, Hong Guo }}{ }^{1,2}$, Juan Qian ${ }^{1,2}$, Aaron Petty ${ }^{1,2}$, James \\ Kaspar $^{1,2}$, Andrew J. Murphy, David M. Valenzuela, George Yancopoulos, Dolores \\ Hambardzumyan ${ }^{4,5}$, Justin D. Lathia ${ }^{4,5}$, Jeremy N. Rich ${ }^{4,5}$, Jeongwu Lee ${ }^{4,5}$, and Bingcheng \\ Wang $1,2,5,{ }^{*}$ \\ ${ }^{1}$ Rammelkamp Center for Research, MetroHealth Campus, and Department of Pharmacology \\ and Oncology, Case Western Reserve University School of Medicine, 2500 MetroHealth Drive, \\ Cleveland, Ohio 44109, USA \\ 2 VelociGene Division, Regeneron Pharmaceuticals, Inc., Tarrytown, NY 10591, USA \\ ${ }^{4}$ Department of Stem Cell Biology and Regenerative Medicine, Lerner Research Institute, \\ Cleveland Clinic, Cleveland, Ohio, 9500 Euclid Ave, Cleveland, Ohio, 44195, USA \\ ${ }^{5}$ Case Comprehensive Cancer Center, School of Medicine, Case Western Reserve University, \\ 11100 Euclid Ave, Cleveland Ohio, 44106, USA
}

\begin{abstract}
Diffuse infiltrative invasion is a major cause for the dismal prognosis of glioblastoma (GBM), but the underlying mechanisms remain incompletely understood. Using human glioblastoma stem cells (GSCs) that recapitulate the invasive propensity of primary GBM, we find that EphA2 critically regulates GBM invasion in vivo. EphA2 was expressed in all seven GSC lines examined, and overexpression of EphA2 enhanced intracranial invasion. The effects required Akt-mediated phosphorylation of EphA2 on serine 897. In vitro the Akt-EphA2 signaling axis is maintained in the absence of ephrin-A ligands and is disrupted upon ligand stimulation. To test whether ephrinAs in tumor microenvironment can regulate GSC invasion, the newly established Efnal;Efna3;Efna4 triple knockout mice (TKO) were used in an ex vivo brain slice invasion assay. We observed significantly increased GSC invasion through the brain slices of TKO mice relative to wild type littermates. Mechanistically EphA2 knockdown suppressed stem properties of GSCs, causing diminished self-renewal, reduced stem marker expression and decreased tumorigenicity. In a subset of GSCs, the reduced stem properties were associated with lower Sox 2 expression. Overexpression of EphA2 promoted stem properties in a kinase-independent manner and
\end{abstract}

Users may view, print, copy, download and text and data- mine the content in such documents, for the purposes of academic research, subject always to the full Conditions of use: http://www.nature.com/authors/editorial_policies/license.html\#terms

*Corresponding Authors: Bingcheng Wang: bxw14@ case.edu Rammelkamp Center for Research/R421 MetroHealth Medical Center Case Western Reserve University 2500 MetroHealth Drive Cleveland, OH 44109 Phone:(216) 778-4256 FAX: (216) 778-4321 Hui Miao: hxm35@ case.edu Rammelkamp Center for Research/R406 MetroHealth Medical Center Case Western Reserve University 2500 MetroHealth Drive Cleveland, OH 44109 Phone:(216)778-8238 FAX: (216) 778-4321.

CONFLICT OF INTEREST

We declare that there are no competing financial interests in relation to the work described by any co-authors of this paper.

SUPPLEMENTARY INFORMATION accompanies the paper on the Oncogene website (http://www.nature.com/onc). 
increased Sox 2 expression. In addition to suppressing invasion, disrupting Akt-EphA2 crosstalk attenuated stem marker expression and neurosphere formation while having minimal effects on tumorigenesis, suggesting that the Akt-EphA2 signaling axis contributes to the stem properties. Taken together, the results show that EphA2 endows invasiveness of GSCs in vivo in cooperation with Akt and contributes to the maintenance of stem properties.

\section{Keywords}

EphA2; Akt; stem cells; glioma; invasion; ephrin-A

\section{INTRODUCTION}

Glioblastoma multiforme or GBM is the most common form of human primary malignant brain tumors (1). Despite maximum treatment including radio- and chemo-therapy, the prognosis of GBM remains dismal with median survival of around 14 months and five year survival only about $3 \%$ (2). A major cause for the lethality is attributed to the diffuse infiltrative invasion of GBM cells throughout the brain at the time of diagnosis, which is responsible for virtually invariable recurrence after surgical resection of primary tumors $(1 ; 3)$. Understanding molecular mechanisms underpinning the highly invasive properties of GBM has significant basic and clinical implications.

Extensive molecular studies have identified phosphatidylinositol 3-kinase (PI3K)/Akt signaling cascade as one of the most frequently altered pathways in primary and secondary GBM (4-6). These observations were further validated and supported by recent results from The Cancer Genome Atlas (TCGA) analyses of large number of GBM cases. It was found that Akt is activated in a great majority ( $88 \%)$ of the GBM cases $(6 ; 7)$, and along with inactivation of $\mathrm{p} 53$ and $\mathrm{Rb}$ pathways represents one of the three major molecular mechanisms of gliomagenesis. Activation of upstream RTK growth factor receptors through amplification, mutation or gene fusion as well as the loss of the negative regulator, PTEN, are the major causes for PI3K/Akt activation, particularly in primary GBM (8-10). However whether and how Akt activation may regulate diffuse infiltrative invasion of GBM remain to be elucidated.

The 14 members of Eph receptor tyrosine kinases and their membrane-anchored ligands called ephrins play important roles in a diverse array of developmental, physiological and disease processes (11-14). Both the receptors and ligands are divided into two subfamilies with EphA receptors primarily binding to GPI-anchored ephrin-A ligands and EphB receptors preferentially engaging the transmembrane ephrin-B ligands (15). Perturbation of Eph/ephrin systems has been documented in many different types of human cancers (16). EphA2 in particular is the most frequently affected Eph receptors in human cancer. In a number of human cancers EphA2 is often overexpressed, which is often associated with the late stage malignant progression and poor prognosis $(16 ; 17)$. While EphA2 is poorly expressed in the normal brain, it is overexpressed in GBM and associated worse prognosis $(18 ; 19)$, making it an attractive target for the development of novel therapeutic strategies $(20 ; 21)$. While many of these studies point to the pro-oncogenic functions of EphA2, 
extensive evidence exist that demonstrate tumor suppressor functions of EphA2 upon ligand stimulation (16;17). Thus ligand-activated EphA2 inhibits Ras/ERK and PI3/Akt pathways, suppresses cell proliferation, and induces apoptosis. Moreover EphA2 knockout mice displayed dramatically increased susceptibility to carcinogenesis (22).

We recently discovered a reciprocal regulatory loop between EphA2 and Akt, which provides a partial mechanistic explanation for the seemingly conflicting roles of EphA2 in cancer (19). It turns out that EphA2 has diametrically opposite functions in regulating cell migration: ligand-dependent inhibition and ligand-independent stimulation. The ligandindependent promotion of cell migration by EphA2 requires Akt-mediated phosphorylation of EphA2 on serine 897 located in the loop between kinase domain and sterile alpha motif (SAM) (19). However, since these studies were performed in vitro, an important unanswered question is whether the Akt-EphA2 signaling axis can regulate tumor cell invasion in vivo.

In this report, we chose to address this outstanding question by using cells at the apex of the cellular hierarchy in GBM (23), glioblastoma stem cells (GSCs). GSCs are responsible for tumor propagation (24-28), therapeutic resistance (29;30), and have a higher invasive capacity (31;32). Here we report that EphA2 signaling axis is required for intracranial invasion of GSCs. We further demonstrate that expression of EphA2 plays a role in maintaining the stem cell properties of GSCs, which is in part mediated through Aktinduced S897 phosphorylation of EphA2. To interrogate invasion in a microenvironment lacking ephrin-A ligands, we utilized brain slices from Efnal;Efna3;Efna4 triple knockout mice and observed significantly increased invasion of GSCs in brain slices from knockout mice compared with those from the wild type or heterozygous littermates, which provides experimental evidence for the long suspected role of ephrins in tumor microenvironment in regulating tumor cells dissemination. Together our data reveal EphA2 as an important driver in the diffuse infiltrative invasion of GBM and help maintain stem properties of GSCs.

\section{RESULTS}

\section{Akt-EphA2 signaling axis is activated in glioma stem cells (GSC)}

To investigate the role of EphA2 in glioma cell invasion in vivo, we utilized the recently established lines of GSCs at early passages, which express multiple stem markers, are capable of self-renewal and differentiation, and form tumors in vivo $(33 ; 34)$. These cells recapitulate the gene expression patterns and in vivo biology of human GBM including diffuse infiltrative invasion of brain parenchyma upon intracranial implantation (33), and therefore constitute a good model to investigate the role of EphA2 in GBM invasion. The GSCs were maintained either in suspension or on laminin-coated surface as monolayer. The latter culturing method is recently shown to maintain stem cell property during in vitro culture and facilitate genetic manipulation (35). Fig. 1A shows neurosphere formation by two lines of GSCs, 827 and 1228, in suspension. Immunofluorescent staining showed that monolayer 827 cells expressed moderate to high levels of endogenous EphA2 (Fig. 1B). Majority of the cells expressed the stem marker nestin, whereas only a small fraction of cells were positive for GFAP, a differentiation marker. Biochemical analysis revealed strong serine 897 phosphorylation (pS897-EphA2) signal (Fig. 1C), demonstrating that the previously characterized ligand-independent Akt-EphA2 signaling axis is active in these 
cells (19). There was little basal tyrosine-phosphorylation in the juxtamembrane domains of Eph receptors (p-EphA/B), indicating a general lack of ligand-induced activation of Eph receptors including EphA2, which was consistent with the undetectable expression of cognate ligands such as ephrin-A1 (Fig. 1C). Stimulation with exogenous ligand ephrin-A1 led to activation of EphA2 and inactivation of Akt, concomitant with dephosphorylation of Akt substrate site S897 (Fig. 1C,D). Consistent with our earlier report in many other cell types (36), ERK1/2 activities were also markedly reduced upon ligand stimulation in GSCs. Therefore, EphA2 receptor is expressed in glioma stem cells, where it mediates liganddependent signaling as evidenced by Akt and ERK inhibition, as well as ligand-independent signaling indicated by S897 phosphorylation.

GSCs are known to share stem properties and transcriptomic signatures with the normal neural stem cells (NSCs) (33). We found that EphA2 is also expressed at significant level in NSCs and mediates Akt inhibition upon ligand stimulation, similar to 827 GSCs (Fig. 1C) and six other GSC preparations (Fig. 1E). Because normal human brain tissues express little EphA2, the fact that it is expressed in all seven GSC lines tested and NSCs indicates a potential role of EphA2 in stem cell regulation, which will be evaluated below.

Like GBM specimens where they originate from, GSCs can be classified into molecularly distinct subtypes (37;38). Among the GSC lines used here, 827, 1228 and 131 bear molecular signatures resembling proneural, classical and mesenchymal subtypes according to TCGA classification scheme (38) (Jeongwu Lee unpublished data). EphA2 expression and ligand-induced tumor suppressive signaling including inhibition of Akt occur in all three subclasses (Fig. 1E), suggesting that EphA2 may be implicated in the pathogenesis of different GBM subclasses. Therefore, loss of ephrin-A ligand expression or engagement with the overexpressed EphA2, as often seen in GBM, is expected to facilitate excessive activation of Akt, which in turn phosphorylates EphA2 on S897, and converts EphA2 from a tumor suppressor to a pro-invasive oncogenic protein.

\section{S897 phosphorylation of EphA2 by Akt promotes GSCs invasion in vivo}

To investigate whether Akt-EphA2 signaling axis may regulate glioma invasion in vivo, we introduced WT- or S897A-EphA2 into 827 and D456MG glioma stem cells via retroviral infection. After brief selection with puromycin, cells were tagged with eGFP or mCherry via lentiviral infection. The sequential viral infections and brief selection did not alter the morphology (Fig. S1A), stem properties of GSCs in term of neurosphere formation and stem marker expression (Fig. S1B,C). Biochemical characterization shows that equivalent expression of WT- or S897A-EphA2 (Fig. 2A). Ephrin-A1 stimulation induced tyrosine phosphorylation of both WT- and S897A-EphA2 and caused their degradation. There was a dramatic increase in basal S897 phosphorylation in WT-EphA2 transduced cells compared with vector control, which became rapidly dephosphorylated upon ligand stimulation. As expected, cells expressing S897A mutant EphA2 showed no increase pS897 (Fig. 2A). Throughout the remainder of the studies, changes in invasive propensities and effects on the stem properties will be made by direct comparisons between control and experimental cells engineered in the identical manner. 
The GFP- and mCherry-tagged 827 cells expressing WT- or S897-EphA2 were mixed at a 1:1 ratio and stereotactically coinjected into NOD/SCID mouse brain. The extent of intracranial invasion was examined 1 - 2 months later on the consecutive coronal brain sections under epifluorescent microscope. The strategy allowed for direct comparison of intracranial invasiveness between the two cell populations on the same sections. We found that the eGFP- tagged WT-EphA2-expressing 827 cells were much more invasive than the mCherry-tagged S897A-EphA2-expressing cells (Fig 2B): While the majority of S897AEphA2-expressing cells remained near the site of injection, the WT-EphA2-expressing cells have diffusely infiltrated the surrounding brain parenchyma, many of them migrating along the corpus callosum to the opposing side of the brain (Fig. 2B). As expected, EphA2 expression driven by the retroviral LTR was retained in the tumor tissues (Fig. S1D).

Similar observations were made using D456MG cells expressing WT- and S897-EphA2 and tagged with GFP and mCherry (Fig. 2C,D). The mCherry-tagged WT-EphA2 cells showed an increased invasion compared with eGFP-tagged S897A-EphA2-expressing cells (Fig. 2D). In addition to the perineural invasion pattern in which the tumor cells invade along the corpus callosum (Fig. 2D left column), WT-EphA2-expressing D456MG cells also exhibited an increased subpial invasion in which the tumor cells migrate under the meninges (Fig. 2D right column). In the third GSC line tested, 1228, more WT-EphA2-expressing cells emerged at distant rostral site than S897A-EphA2-expressing cells (Fig. S2).

The studies above compared relative invasiveness of GSCs expressing WT vs. S897A mutant EphA2. To further examine a possible role of EphA2 in driving GSC cell invasion in vivo, we interrogated if overexpression of wild type EphA2 alone could promote invasion, as the overexpressed EphA2 is known to increase ligand-independent Akt-EphA2 crosstalk and stimulate cell migration and invasion in vitro (19). We chose 1228 cells for this study because they expressed lower level of endogenous EphA2. As shown in Fig. S3, EphA2 overexpression in 1228 cells elevated perineural and subpial invasion relative to vector control cells, demonstrating that elevated EphA2 expression can indeed promote GSC invasion in vivo.

In aggregate, these results demonstrate that Akt-EphA2 signaling axis is active in glioma stem cells, and is required for infiltrative invasion of glioma stem cells in vivo. Given the overexpression of EphA2 and activation of Akt in the vast majority of human GBM $(4 ; 6 ; 21)$, Akt-EphA2 crosstalk likely constitute an important driver mechanism in promoting GBM invasion with deadly consequences.

\section{Ephrin-As in brain microenvironment provide major repulsive guidance cues for infiltrative invasion of GSC}

- The best characterized function of Eph/ephrin interactions in vivo is the repulsive guidance of growth cone and cell migration (39). However, an important question remains whether ephrins in the tumor microenvironment can similarly repulse invading tumor cells in vivo. In vitro the pro-migratory and pro-invasive Akt-EphA2 signaling axis is activated in the absence of ephrin-A ligands. In fact, ligand-induced activation of EphA2 inhibits Akt activities, severs the Akt-EphA2 axis, and suppresses cell migration (19). Therefore we hypothesize that a tumor microenvironment that lacks ephrin-A ligands would not only 
remove repulsive cues in the tumor microenvironment, but also accelerate glioma dispersal by facilitating ligand-independent pro-invasive function of EphA2.

There are five Efna genes in mammalian systems encoding five distinct ephrin-A ligands. Interestingly, three of them (Efnal, Efna3 and Efna4) reside on the same region of mouse chromosome 3 in a genomic sequence of about 72kb (Fig. 3A). No other genes are found in this region in the existing databases. The expression of the three genes in the brain was confirmed from single gene knockouts for Efnal, Efna3 or Efna4 genes (Fig. 3B). Both Efnal and Efna3 genes are highly expressed in E15.5 and adult brains with significant overlapping patterns as revealed by staining for the expression of $L a c Z$ knockin cassette. Expression of Efna4 is lower but detectable at higher power (Fig. 3B). All three ephrin-As could contribute to the regulation of tumor cell invasion due to their similar ability to bind and activate EphA2. Simultaneous knockout of all three genes by deleting the entire genomic region will enable the assessment of how three ephrin-As can collectively affect in vivo invasion. Toward this end, we utilized the VelociGene technology developed at Regeneron as part of the NIH-sponsored international consortium to systemically knockout all mouse genes (40). Successful deletion of the entire region led to establishment of Efnal;Efna3;Efna4 triple knockout (TKO) mice, providing an unprecedented powerful platform to investigate the roles of EphA/ephrin-A in physiology and diseases. To confirm the complete knockout of all three Efna genes, quantitative real time PCR (qRT-PCR) was performed on RNA extracted from different regions of adult brains of TKO and heterozygous knockout mice. Fig. 3C shows that expression of Efnal, Efna3 and Efna4 expression expressed various levels in heterozygous brain were completely absent from the TKO mice. Moreover there was no compensatory upregulation of Efna2 and Efna5 expression in all the regions examined. Phenotypically the TKO mice appear to be normal and are fertile once they are born, although there are fewer TKO pups than expected from the Mendelian ratios. Further studies are needed to find causes for embryonic lethality.

To assess how ephrin-As in brain microenvironment may affect invasion of human GSCs, brain slices were prepared from TKO mice (Efnal/a3/a4-/-) and WT or heterozygous littermates (Efnal/a3/a4+/+or +/-) (Fig. 3D). The 827 GSCs tagged with eGFP were implanted in the cortical region near the corpus callosum. Images taken at 3 hours after implantation, when the cell had just settled, were used as starting points (Fig. 3D). Twentyfour hours after implantation, 827 cells already spread out from the implantation sites in the TKO mouse brain slices. In contrast, much less invasion was observed in WT brain slices (Fig. 3D). Quantitative analyses of the areas covered by the invading cells show significant difference in invasion in heterozygous vs. TKO brain slices (Fig. 3E). Similar results were obtained with mCherry-tagged 1228 GSCs (Fig. S4). The fact that deletion of three ephrin-A ligands leads to increased invasiveness of GSC provides strong support for a major role of ephrin-As in the repulsive guidance of invading glioma cells in the brain microenvironment. The data also suggest that the pro-invasive Akt-EphA2 signaling axis can be overridden by the ligands, and may force the invading GBM cells to choose paths less decorated with ephrin-As.

In addition to accelerated invasiveness, we also noticed an increase in tumor cell mass in TKO brain slices compared with those from WT or heterozygous littermates. This is evident 
in the circled areas in Fig. 3D,F and Fig. S4,S5, where significant expansion of tumor cell mass consistently occurred in TKO brain slices between 3 and 48 hours, whereas only minor changes were observed in WT or heterozygous brain slices. Ligand-induced EphA2 activation strongly inhibited ERK1/2 activities in GSCs (Fig. 1B) similar to many other cell types (36). In mouse skin carcinogenesis model in vivo and human prostate cancer cells in vitro, EphA2-mediated suppression of ERK is linked to tumor growth (22) and cell proliferation (36). Therefore it is possible that deletion of Efnal/a3/a4 could significantly reduce the growth suppressive effects through the ligand-activated EphA2 or other EphA receptors, leading to increased GSC growth, although reduced apoptosis could also be a contributing mechanism. Further investigation is needed to delineate the exact mechanisms.

\section{Silencing EphA2 expression inhibits tumor development and invasion}

Next, we examined how knocking down EphA2 expression could impact invasion in vivo. Immunoblot showed a significant reduction of EphA2 expression following shRNAknockdown in 827 GSCs compared with control (Fig. 4A). In the modified Boyden chamber cell migration assay, EphA2 knockdown resulted in reduced chemotactic migration toward bFGF and EGF (Fig. S6). To investigate how downregulation of EphA2 affects the tumor behavior in vivo, the mCherry- or eGFP-tagged knockdown and control cells were coinjected into NOD/SCID mouse brain. Surprisingly, knocking down EphA2 in 827 cells markedly reduced the intracranial tumor development in vivo. While a big tumor mass was generated from eGFP-tagged control cells, only a small number of mCherry-tagged EphA2 shRNA-expressing were found in the NOD/SCID mouse brain (Fig 4B), despite the same number of cells injected. The effects were apparently cell context dependent, as D456MG cells with similar EphA2 knockdown (Fig. 4C) were still tumorigenic, although the tumor was smaller than the control at the injection site (Fig. 4D, left panel). The retention of tumorigenicity of D456MG-shEphA2 cells allowed assessment of EphA2 knockdown on invasion. As shown in Fig. 4D, whereas vector control D456MG cells exhibited extensive local and distal dispersal, cells with EphA2 knockdown showed reduced ability to invade locally and disseminate distally (Fig. 4D), further supporting a role of EphA2 in driving glioma invasion in vivo.

\section{EphA2 helps maintain stem properties of GSCs in part through crosstalk with Akt}

The dramatically reduced tumorigenicity of 827 cells following EphA2 knockdown suggested that EphA2 may play a role in maintaining stem properties in a subset of GSCs. To test this possibility, we analyzed the expression of the known stem cell and differentiation makers in 827 cells expressing EphA2 shRNA. Among the markers examined, we found that downregulation of EphA2 significantly reduced the expression of Nestin and Sox 2 and increased the expression of GFAP (Fig. 5A). Next we performed clonogenicity assay where the capacity of neurosphere formation is examined following limiting dilution, a widely accepted and sensitive in vitro assay for determining the selfrenewal capacity of GSCs in vitro. Consistent with the changes in stem and differentiation marker expression, the ability of 827 cells to form neurospheres was reduced by about 4 folds upon lentiviral shRNA-mediated silencing of EphA2 expression (Fig. 5B). Even when the neurospheres did form, they were significantly smaller than those from the cells transduced with control lentiviral vector (Fig. 5C). Thus EphA2 knockdown caused 
reductions in both the number and sizes of neurosphere formation. Together with the marked reduction in tumorigenicity, the data together support a role of EphA2 in maintaining stem properties of GSCs.

Conversely overexpression of EphA2 led to a three-fold increase in neurosphere formation (Fig. 6A). The increase in GSCs self-renewal is dependent on the Akt-EphA2 signaling axis, as cells expressing S897A mutant EphA2 failed to promote neurosphere formation (Fig. 6A). Overexpression of S897A-EphA2 did not show significant effects on the sizes of neurospheres either (Fig. 6B), suggesting that the endogenous EphA2 is sufficient to support basal level of GSCs self-renewal similar to vector control cells (Fig. 5A,B). These results were further validated with 1228 and D456MG cells where overexpression of WT EphA2 but not S897A mutant EphA2 significantly increased number of neurospheres (Fig. S7). Interestingly the tyrosine kinase-dead mutant (D739N) of EphA2 retained the ability to promote self-renewal capacity, suggesting that the effects were mediated by the kinaseindependent mechanisms (Fig. 6C).

Glioma stem cells maintain stemness using similar mechanisms as normal neural stem cells and embryonic stem cells. The down regulation of Sox2 upon silencing of EphA2 expression (Fig. 5A) is of particular interest as Sox 2 is critical for stem property maintenance $(41 ; 42)$. Recent studies show that the expression of Sox 2 is elevated with increasing grades of gliomas (43;44). Silencing of Sox 2 resulted in the loss of tumorigenicity of glioma stem cells (45). To determine whether Akt-EphA2 crosstalk also regulates the expression of Sox2, we examined the Sox 2 in 827 cells that overexpress WT- or S897AEphA2. Consistent with the observation that silencing EphA2 expression in 827 cells reduced the expression of Sox 2 (Fig. 5A), overexpression of EphA2 in these cells resulted in an increased expression of Sox2. Interestingly the increase in Sox 2 level was largely abolished by S897A mutation (Fig. 6D,E). Similar results were obtained in 1228 cells (Fig. $6 \mathrm{~F}, \mathrm{G})$. Together these data suggest that EphA2 receptor regulates the expression of Sox 2 through crosstalk with Akt, which may contribute to the maintenance of GSC stem properties.

In sum, several lines of evidence presented here suggest that Akt-EphA2 signaling axis contribute to the stem properties including: 1) significantly diminished intracranial invasiveness of cells expressing S897A EphA2 relative to WT-EphA2, and invasiveness is often linked to EMT and stem properties (Fig. 2) (46), 2) reduced ability of S897A mutant EphA2 to regulate Sox2 expression compared with WT-EphA2 (Fig. 6), and 3) impaired neurosphere formation by S897A-EphA2 compared with WT-EphA2 (Fig. 6 and S6). However, unlike the EphA2 knockdown, the S897A mutation did not significantly affect tumorigenesis (Fig. 2). Additional yet to be identified mechanisms may contribute to the maintenance of stem properties by EphA2.

\section{DISCUSSION}

In this report we provide multiple lines of evidence demonstrating Akt-EphA2 signaling axis as a driving mechanism underlying invasive propensity of GBM in vivo, a major cause of the poor prognosis of the disease. EphA2 knockdown markedly suppressed both self- 
renewal and intracranial tumorigenicity, demonstrating a role of EphA2 in the maintenance of glioma stem properties. S897A mutation of EphA2 affected self renewal and stem marker expression, but did not have significant effects on tumorigenesis, suggesting that crosstalk with Akt is partially responsible, but other yet unidentified mechanisms are likely at play as well. Finally the long suspected role of ephrins in tumor microenvironment in regulating tumor cell dissemination is experimentally illustrated using the novel Efnal;Efna3;Efna4 triple knockout mouse model, highlighting the complex nature of tumor invasion in vivo that can be dictated not only by cell autonomous mechanisms but also by ephrin-As in the tumor microenvironment.

The propensity for diffuse infiltrative invasion throughout the brain is a defining hallmark of GBM. The molecular mechanisms that drive the rampant GBM invasion in vivo are not fully understood. In spite of the ongoing debate on their existence and prevalence, the discovery of cancer stem cells (CSCs) has provided a valuable platform for understanding the cellular and molecular heterogeneity of tumors. Increasing evidence suggest that tumor cell stemness is associated with aggressive tumor behaviors relevant to tumor dissemination and metastasis such as tumor cell motility, invasiveness and increased resistance to apoptosis (47). We show here that the Akt-EphA2 signaling axis may contribute to invasive propensities of GSCs in part by regulating their stem properties. In GSC lines 827 and 1228 derived from adult glioblastomas, but not D456MG derived from childhood glioma, Sox 2 levels were elevated by overexpression of WT- but not S897A-EphA2. Nevertheless, in all three lines of GSCs, WT- but not S897AEphA2 promotes the ability of cells to form neurospheres. These results suggest that there exist different molecular mechanisms downstream from Akt-EphA2 axis to promote self-renewal in different GSCs or cellular context. The current observation is in keeping with our previous report showing that high level of pS897-EphA2 occurred in the perivascular regions of human glioblastoma specimens (19), a place that has recently been identified as brain tumor stem cell niche (48). Further characterization of stem cell regulation by Akt-EphA2 signaling axis can lead to new insights on GSC maintenance and identification of new points of therapeutic intervention for GBM.

The repulsive guidance of cell migration is one of the most well-established functions of Eph/ephrin bidirectional signaling upon cell-cell contact (14), which has led to the suggestions that ephrins presented by cells in tumor microenvironment could negatively regulate tumor cell dissemination (49). However the experimental proof has remained elusive. The present report provides the first experimental evidence that ephrin-A1, -A3, and -A4 in normal brain indeed have repulsive role in regulating glioma cell invasion. Since there are five known ephrin-As, all of which are expressed in the brain, it is quite possible that the remaining ephrin-A2, and -A5 could compensate in some way for the loss of ephrin-A1, -A3, and -A4. While obtaining a complete ephrin-A-null model is now feasible, our current triple knockout mice provides a proof that ephrin-As expressed on cells along the invasive path of glioma cells suppress glioma invasion. Much like growth cones in nervous development, the invading tumor cells in vivo may be forced to choose routes lined with cells less decorated with ephrin-As. This may in turn govern the pattern and degree of GBM invasion in vivo. Importantly our data also suggest that mis-regulation ephrin-A 
expression in tumor microenvironment is likely to have profound impact on GBM cell invasion. In this regard, the recently discovered monomeric ephrin-A1 resulting from proteolytic cleavage of cell surface ephrin-A1 in vitro and GBM tissues in vivo could also potentially regulate GBM invasion (50;51).

Along this line, in both glioma stem cells (shown in this report) and established glioma cells lines (19), EphA2 can be readily activated by exogenous ligand ephrin-A1, leading to the inhibition of both Akt and Erk1/2 activities. A recent report suggests that Erk1/2 are more active in glioma core and less active at invasive front; whereas Akt is more active at invasive front where tumor cells gain stem properties (52). EphA2 activation by ligand stimulation is capable of inhibiting both Erk1/2 and Akt, suggesting that targeting EphA2 could potentially be beneficial for both primary and invasive tumor cells. Toward this end, the newly discovered small molecule agonists for EphA2 (53) and other agents targeting Eph/ephrin system at different stages of development (16) may prove useful in therapeutic intervention of GBM.

While this manuscript was under revision, a report by Binda et al. provides evidence that EphA2 is selectively upregulated in tumor propagating cells in human GBM, and knockdown of EphA2 suppressed self renewal (54). Consistent with the dual liganddependent tumor suppressor and ligand-independent oncogenic function of EphA2 (19), administration of ephrin-A1 suppressed gliomagenesis of intracranially implanted human GSCs. The authors also demonstrate that S897-phosphorylated EphA2 is associated with stem cell populations, although the effects of $\mathrm{S} 897$ phosphorylation on stem properties and invasion were not directly investigated. The regulatory roles of Eph receptors in the maintenance glioma stem cells are not limited to EphA2. Similar roles have also been attributed to EphB2 (55) and most recently EphA3 (56). Together with the results presented here, the convergence of evidence suggests that the Eph/ephrin systems, aside from their well-established indispensible functions in the developing nervous system, are essential regulators of etiology and invasion of GBM, and represent exciting targets in developing novel therapies for the disease.

\section{MATERIALS AND METHODS}

\section{Antibodies and reagents}

Rabbit polyclonal anti-pS374-Akt, anti-Akt, and anti-GAPDH were purchased from Cell Signaling. Rabbit anti-p-EphA/B was raised against the conserved phosphopeptides from the juxtamembrane regions. Rabbit polyclonal phospho-S897-specific antibody was raised against phospho-peptide DPRVSIRLP-pS ${ }^{897}$-TSGSEGVPFR and has been characterized previously (19). Rabbit polyclonal anti-EphA2, anti-ephrin-A1, anti-ERK1, mouse monoclonal anti-p-ERK1/2 were purchased from Santa Cruz Biotechnology. Mouse monoclonal anti-EphA2 was a kind gift from Dr. Andrew Boyd (The University of Queensland, Australia). Mouse monoclonal anti-Sox2 was purchased from R\&D System. Rabbit polyclonal anti-Nestin were purchased from Abcam. Mouse monoclonal anti-GFAP was purchased from Sigma. Ephrin-A1-Fc was produced as described (49). 


\section{Human glioblastoma stem cell culture and viral infection}

Human GSCs were kindly provided by Dr. Fine (NIH-NCI) and cultured as described previously (33). For retroviral or lentiviral infection, GSCs were seeded on laminin-coated dishes and infected with virus produced in neurobasal medium. The stable cell lines were obtained by selecting the infected cells for puromycin-resistance. GSCs that stably express WT-EphA2, S897A-EphA2, EphA2shRNA, non-silencing shRNA control, or vector control were further infected with lentivirus expressing mCherry or eGFP.

\section{In vivo studies}

All animal experiments were carried out according to approved protocols from the Institutional Animal Care and Use Committee (IACUC) at Case Western Reserve University. To examine the intracranial invasiveness of glioma stem cells, Total of $2 \times 10^{5}$ eGFP-and mCherry-tagged cells (1:1 ratio) were injected into the lateral ventricles of P2 NOD/SCID mice. Mice were sacrificed 2 weeks (for 1228 cells), 1 month (for D456MG cells) or 2 months (for 827 cells) post injection. Mouse brains were removed and fixed in $4 \%$ paraformaldehyde. Consecutive coronal sections were cut from the sucrose-processed frozen brains. The sections were examined under fluorescent microscope. Images were taken using Leica DM-IRE2 microscope operated from MetaMorph 7.0.

\section{Brain slice invasion assay}

Efnal/a3/a4 triple KO (TKO) neonatal mice, wild-type, or heterozygous littermates were sacrificed and the brains were dissected out. The brain was embedded in the $1 \%$ agrose gel and $300 \mu \mathrm{m}$ brain slices were cut using vibratome (Leica VT 1000P) in ice-cold PBS containing $2.5 \%$ glucose. The brain slices were cultured on the Transwell inserts $(0.4 \mu \mathrm{m}$ pore size, BD Biosciences) in 6-well plate containing MEM/15\% horse serum and allowed to equilibrate overnight in $37^{\circ} \mathrm{C} / 5 \% \mathrm{CO} 2$ incubator. GSC 827 -eGFP or 1228-WT-EphA2mCherry $\left(2 \times 10^{3}\right.$ cells in $0.04 \mu \mathrm{l}$ or $1 \times 10^{4}$ cells in $\left.0.5 \mu \mathrm{l}\right)$ were implanted in the cortical region near the corpus callosum. Images were taken 3, 24, 48, and 72 hours after cell implantation using Leica DM-IRE2 microscope operated from MetaMorph 7.0.

\section{Immunofluorecence}

Glioma stem cells were seeded on laminin-coated coverslips and cultured for overnight. Cells were fixed with 4\% EM-grade paraformaldehyde and stained with rabbit anti-Nestin and mouse anti-EphA2 or mouse anti-GFAP. FITC- or Rhodamine red X-Donkey secondary antibodies were used to detect the primary antibody-antigen complex.

\section{Neurosphere formation assay}

Glioma stem cells were dissociated with Accutase and DNases1. One hundred or 1000 GSCs were plated in each well of ultra-low-adhesive clustered tissue culture plates in triplicates for 6-well plates. The numbers of the spheres were counted 4 days later. The images were taken by using Leica DM-IRE2 microscope operated from MetaMorph 7.0. The assay was repeated at least twice by different researchers. For D456MG cells, 1 or 10 cells were seeded in 96-well ultra-low-adhesive clustered tissue culture plates. The numbers of wells that have sphere formation were counted. 


\section{Supplementary Material}

Refer to Web version on PubMed Central for supplementary material.

\section{ACKNOWLEDGEMENT}

This work was supported by grants from National Institute of Health CA152371 (H.M. \& B.W.), and by awards to B.W. from FAMRI Foundation (072216_CIA) and Prayers From Maria Foundation. This research was also supported by the Case Comprehensive Cancer Center (P30 CA43703).

\section{REFERENCES}

1. Furnari FB, Fenton T, Bachoo RM, Mukasa A, Stommel JM, Stegh A, et al. Malignant astrocytic glioma: genetics, biology, and paths to treatment. Genes Dev. Nov 1; 2007 21(21):2683-710. [PubMed: 17974913]

2. Stupp R, Hegi ME, Mason WP, van den Bent MJ, Taphoorn MJ, Janzer RC, et al. Effects of radiotherapy with concomitant and adjuvant temozolomide versus radiotherapy alone on survival in glioblastoma in a randomised phase III study: 5-year analysis of the EORTC-NCIC trial. Lancet Oncol. May; 2009 10(5):459-66. [PubMed: 19269895]

3. Chakravarti A, Palanichamy K. Overcoming therapeutic resistance in malignant gliomas: current practices and future directions. Cancer Treat Res. 2008; 139:173-89. [PubMed: 18236717]

4. Dunn GP, Rinne ML, Wykosky J, Genovese G, Quayle SN, Dunn IF, et al. Emerging insights into the molecular and cellular basis of glioblastoma. Genes Dev. Apr 15; 2012 26(8):756-84. [PubMed: 22508724]

5. Li J, Yen C, Liaw D, Podsypanina K, Bose S, Wang SI, et al. PTEN, a putative protein tyrosine phosphatase gene mutated in human brain, breast, and prostate cancer [see comments]. Science. 1997; 275(5308):1943-7. [PubMed: 9072974]

6. TCGA Research Network. Comprehensive genomic characterization defines human glioblastoma genes and core pathways. Nature. Sep 4.2008 455:1061-8. [PubMed: 18772890]

7. Parsons DW, Jones S, Zhang X, Lin JC, Leary RJ, Angenendt P, et al. An Integrated Genomic Analysis of Human Glioblastoma Multiforme. Science. Sep 4.2008 321:1807-12. [PubMed: 18772396]

8. Dunn GP, Rinne ML, Wykosky J, Genovese G, Quayle SN, Dunn IF, et al. Emerging insights into the molecular and cellular basis of glioblastoma. Genes Dev. Apr 15; 2012 26(8):756-84. [PubMed: 22508724]

9. Frattini V, Trifonov V, Chan JM, Castano A, Lia M, Abate F, et al. The integrated landscape of driver genomic alterations in glioblastoma 4. Nat Genet. Aug 5.2013

10. Singh D, Chan JM, Zoppoli P, Niola F, Sullivan R, Castano A, et al. Transforming fusions of FGFR and TACC genes in human glioblastoma 17. Science. Sep 7; 2012 337(6099):1231-5. [PubMed: 22837387]

11. Kullander K, Klein R. Mechanisms and functions of Eph and ephrin signalling. Nat Rev Mol Cell Biol. Jul; 2002 3(7):475-86. [PubMed: 12094214]

12. Miao H, Wang B. Eph/ephrin signaling in epithelial development and homeostasis. Int J Biochem Cell Biol. Apr; 2009 41(4):762-70. [PubMed: 18761422]

13. Pasquale EB. Eph-ephrin bidirectional signaling in physiology and disease. Cell. Apr 4; 2008 133(1):38-52. [PubMed: 18394988]

14. Poliakov A, Cotrina M, Wilkinson DG. Diverse roles of eph receptors and ephrins in the regulation of cell migration and tissue assembly. Dev Cell. Oct; 2004 7(4):465-80. [PubMed: 15469835]

15. Eph Nomenclature Committee. Unified nomenclature for Eph family receptors and their ligands, the ephrins. Eph Nomenclature Committee [letter] [In Process Citation]. Cell. 1997; 90(3):403-4. [PubMed: 9267020]

16. Pasquale EB. Eph receptors and ephrins in cancer: bidirectional signalling and beyond. Nat Rev Cancer. Mar; 2010 10(3):165-80. [PubMed: 20179713] 
17. Miao H, Wang B. EphA receptor signaling--complexity and emerging themes. Semin Cell Dev Biol. Feb; 2012 23(1):16-25. [PubMed: 22040915]

18. Wykosky J, Gibo DM, Stanton C, Debinski W. EphA2 as a novel molecular marker and target in glioblastoma multiforme. Mol Cancer Res. Oct; 2005 3(10):541-51. [PubMed: 16254188]

19. Miao H, Li D-Q, Mukherjee A, Guo H, Petty A, Basilion J, et al. EphA2 Mediates LigandDependent Inhibition and Ligand-Independent Promotion of Cell Migration and Invasion via a Reciprocal Regulatory Loop with Akt. Cancer Cell. 2009; 16:9-20. [PubMed: 19573808]

20. Wykosky J, Gibo DM, Debinski W. A novel, potent, and specific ephrinA1-based cytotoxin against EphA2 receptor expressing tumor cells. Mol Cancer Ther. Dec; 2007 6(12 Pt 1):3208-18. [PubMed: 18089715]

21. Wykosky J, Debinski W. The EphA2 receptor and ephrinA1 ligand in solid tumors: function and therapeutic targeting. Mol Cancer Res. Dec; 2008 6(12):1795-806. [PubMed: 19074825]

22. Guo H, Miao H, Gerber L, Singh J, Denning MF, Gilliam AC, et al. Disruption of EphA2 receptor tyrosine kinase leads to increased susceptibility to carcinogenesis in mouse skin. Cancer Res. Jul 15; 2006 66(14):7050-8. [PubMed: 16849550]

23. Venere M, Fine HA, Dirks PB, Rich JN. Cancer stem cells in gliomas: identifying and understanding the apex cell in cancer's hierarchy. GLIA. Aug; 2011 59(8):1148-54. [PubMed: 21547954]

24. Singh SK, Clarke ID, Terasaki M, Bonn VE, Hawkins C, Squire J, et al. Identification of a cancer stem cell in human brain tumors. Cancer Res. Sep 15; 2003 63(18):5821-8. [PubMed: 14522905]

25. Singh SK, Hawkins C, Clarke ID, Squire JA, Bayani J, Hide T, et al. Identification of human brain tumour initiating cells. Nature. Nov 18; 2004 432(7015):396-401. [PubMed: 15549107]

26. Ignatova TN, Kukekov VG, Laywell ED, Suslov ON, Vrionis FD, Steindler DA. Human cortical glial tumors contain neural stem-like cells expressing astroglial and neuronal markers in vitro. GLIA. Sep; 2002 39(3):193-206. [PubMed: 12203386]

27. Galli R, Binda E, Orfanelli U, Cipelletti B, Gritti A, De VS, et al. Isolation and characterization of tumorigenic, stem-like neural precursors from human glioblastoma. Cancer Res. Oct 1; 2004 64(19):7011-21. [PubMed: 15466194]

28. Hemmati HD, Nakano I, Lazareff JA, Masterman-Smith M, Geschwind DH, Bronner-Fraser M, et al. Cancerous stem cells can arise from pediatric brain tumors. Proc Natl Acad Sci U S A. Dec 9; 2003 100(25):15178-83. [PubMed: 14645703]

29. Bao S, Wu Q, McLendon RE, Hao Y, Shi Q, Hjelmeland AB, et al. Glioma stem cells promote radioresistance by preferential activation of the DNA damage response. Nature. Dec 7; 2006 444(7120):756-60. [PubMed: 17051156]

30. Liu G, Yuan X, Zeng Z, Tunici P, Ng H, Abdulkadir IR, et al. Analysis of gene expression and chemoresistance of CD133+ cancer stem cells in glioblastoma. Mol Cancer. 2006; 5:67. [PubMed: 17140455]

31. Cheng L, Wu Q, Guryanova OA, Huang Z, Huang Q, Rich JN, et al. Elevated invasive potential of glioblastoma stem cells. Biochem Biophys Res Commun. Mar 25; 2011 406(4):643-8. [PubMed: 21371437]

32. Garcia JL, Perez-Caro M, Gomez-Moreta JA, Gonzalez F, Ortiz J, Blanco O, et al. Molecular analysis of ex-vivo CD133+ GBM cells revealed a common invasive and angiogenic profile but different proliferative signatures among high grade gliomas. BMC Cancer. 2010; 10:454. [PubMed: 20735813]

33. Lee J, Kotliarova S, Kotliarov Y, Li A, Su Q, Donin NM, et al. Tumor stem cells derived from glioblastomas cultured in bFGF and EGF more closely mirror the phenotype and genotype of primary tumors than do serum-cultured cell lines. Cancer Cell. May; 2006 9(5):391-403. [PubMed: 16697959]

34. Lee J, Son MJ, Woolard K, Donin NM, Li A, Cheng CH, et al. Epigenetic-mediated dysfunction of the bone morphogenetic protein pathway inhibits differentiation of glioblastoma-initiating cells. Cancer Cell. Jan; 2008 13(1):69-80. [PubMed: 18167341]

35. Pollard SM, Yoshikawa K, Clarke ID, Danovi D, Stricker S, Russell R, et al. Glioma stem cell lines expanded in adherent culture have tumor-specific phenotypes and are suitable for chemical and genetic screens. Cell Stem Cell. Jun 5; 2009 4(6):568-80. [PubMed: 19497285] 
36. Miao H, Wei BR, Peehl DM, Li Q, Alexandrou T, Schelling JR, et al. Activation of EphA receptor tyrosine kinase inhibits the Ras/MAPK pathway. Nat Cell Biol. May; 2001 3(5):527-30. [PubMed: 11331884]

37. Gu C, Banasavadi-Siddegowda YK, Joshi K, Nakamura Y, Kurt H, Gupta S, et al. Tumor-specific activation of the C-JUN/MELK pathway regulates glioma stem cell growth in a p53-dependent manner. Stem Cells. May; 2013 31(5):870-81. [PubMed: 23339114]

38. Verhaak RG, Hoadley KA, Purdom E, Wang V, Qi Y, Wilkerson MD, et al. Integrated genomic analysis identifies clinically relevant subtypes of glioblastoma characterized by abnormalities in PDGFRA, IDH1, EGFR, and NF1. Cancer Cell. Jan 19; 2010 17(1):98-110. [PubMed: 20129251]

39. Wilkinson DG. Multiple roles of EPH receptors and ephrins in neural development. Nat Rev Neurosci. Mar; 2001 2(3):155-64. [PubMed: 11256076]

40. Valenzuela DM, Murphy AJ, Frendewey D, Gale NW, Economides AN, Auerbach W, et al. Highthroughput engineering of the mouse genome coupled with high-resolution expression analysis. Nat Biotechnol. Jun; 2003 21(6):652-9. [PubMed: 12730667]

41. Wegner M. SOX after SOX: SOXession regulates neurogenesis. Genes Dev. Dec 1; 2011 25(23): 2423-8. [PubMed: 22156204]

42. Pevny LH, Nicolis SK. Sox2 roles in neural stem cells. Int J Biochem Cell Biol. Mar; 2010 42(3): 421-4. [PubMed: 19733254]

43. Holmberg J, He X, Peredo I, Orrego A, Hesselager G, Ericsson C, et al. Activation of neural and pluripotent stem cell signatures correlates with increased malignancy in human glioma. PLoS One. 2011; 6(3):e18454. [PubMed: 21483788]

44. Annovazzi L, Mellai M, Caldera V, Valente G, Schiffer D. SOX2 expression and amplification in gliomas and glioma cell lines. Cancer Genomics Proteomics. May; 2011 8(3):139-47. [PubMed: 21518820]

45. Gangemi RM, Griffero F, Marubbi D, Perera M, Capra MC, Malatesta P, et al. SOX2 silencing in glioblastoma tumor-initiating cells causes stop of proliferation and loss of tumorigenicity. Stem Cells. Jan; 2009 27(1):40-8. [PubMed: 18948646]

46. Mani SA, Guo W, Liao MJ, Eaton EN, Ayyanan A, Zhou AY, et al. The epithelial-mesenchymal transition generates cells with properties of stem cells. Cell. May 16; 2008 133(4):704-15. [PubMed: 18485877]

47. Chaffer CL, Weinberg RA. A perspective on cancer cell metastasis. Science. Mar 25; 2011 331(6024):1559-64. [PubMed: 21436443]

48. Calabrese C, Poppleton H, Kocak M, Hogg TL, Fuller C, Hamner B, et al. A perivascular niche for brain tumor stem cells. Cancer Cell. Jan; 2007 11(1):69-82. [PubMed: 17222791]

49. Miao H, Burnett E, Kinch M, Simon E, Wang B. Activation of EphA2 kinase suppresses integrin function and causes focal-adhesion-kinase dephosphorylation. Nat Cell Biol. Feb; 2000 2(2):62-9. [PubMed: 10655584]

50. Beauchamp A, Lively MO, Mintz A, Gibo D, Wykosky J, Debinski W. EphrinA1 is released in three forms from cancer cells by matrix metalloproteases. Mol Cell Biol. Aug; 2012 32(16):325364. [PubMed: 22688511]

51. Wykosky J, Palma E, Gibo DM, Ringler S, Turner CP, Debinski W. Soluble monomeric EphrinA1 is released from tumor cells and is a functional ligand for the EphA2 receptor. Oncogene. Dec 11; 2008 27(58):7260-73. [PubMed: 18794797]

52. Molina JR, Hayashi Y, Stephens C, Georgescu MM. Invasive glioblastoma cells acquire stemness and increased Akt activation. Neoplasia. Jun; 2010 12(6):453-63. [PubMed: 20563248]

53. Petty A, Myshkin E, Qin H, Guo H, Miao H, Tochtrop GP, et al. A Small Molecule Agonist of EphA2 Receptor Tyrosine Kinase Inhibits Tumor Cell Migration In Vitro and Prostate Cancer Metastasis In Vivo. PLoS ONE. Aug 15.2012 7(8):e42120. [PubMed: 22916121]

54. Binda E, Visioli A, Giani F, Lamorte G, Copetti M, Pitter KL, et al. The EphA2 receptor drives self-renewal and tumorigenicity in stem-like tumor-propagating cells from human glioblastomas. Cancer Cell. Dec 11; 2012 22(6):765-80. [PubMed: 23238013]

55. Johnson RA, Wright KD, Poppleton H, Mohankumar KM, Finkelstein D, Pounds SB, et al. Crossspecies genomics matches driver mutations and cell compartments to model ependymoma. Nature. Jul 29; 2010 466(7306):632-6. [PubMed: 20639864] 
56. Day BW, Stringer BW, Al-Ejeh F, Ting MJ, Wilson J, Ensbey KS, et al. EphA3 Maintains Tumorigenicity and Is a Therapeutic Target in Glioblastoma Multiforme. Cancer Cell. Feb 11; 2013 23(2):238-48. [PubMed: 23410976] 
A

C

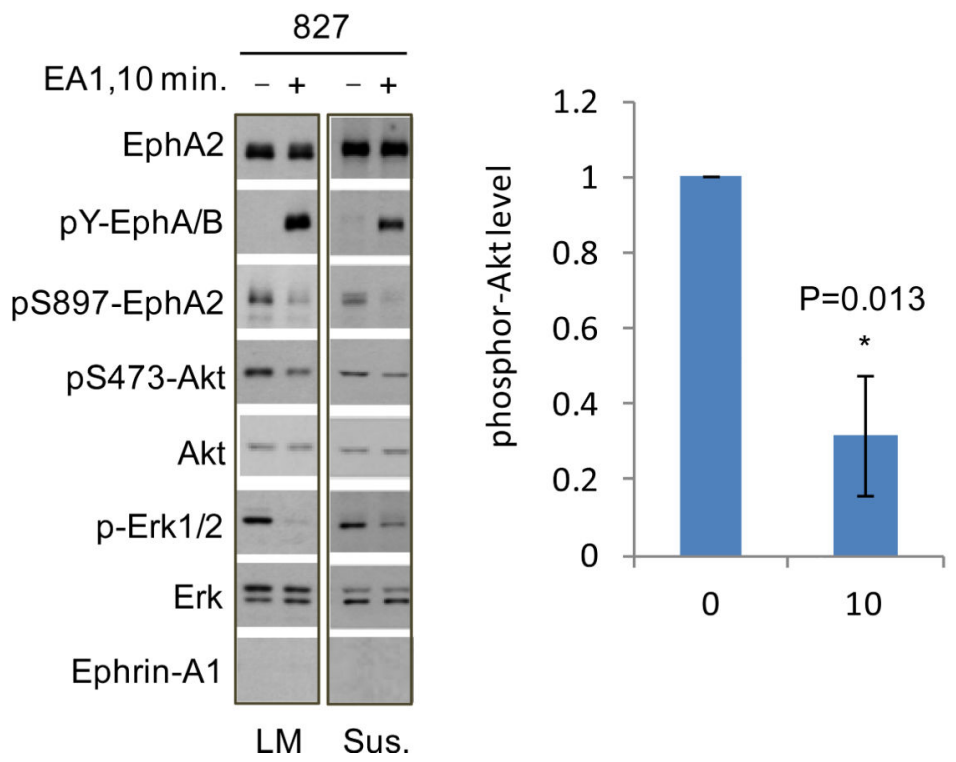

B
827

1228
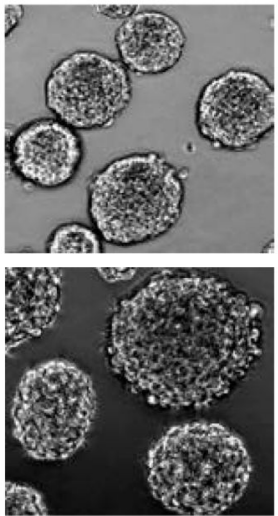

Suspension
Laminin

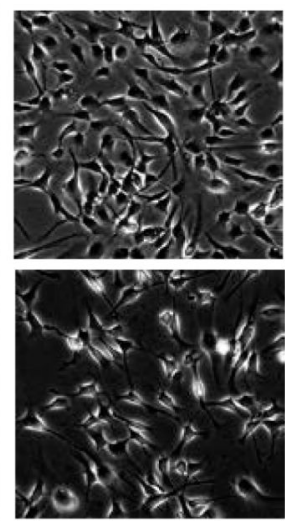

Nestin
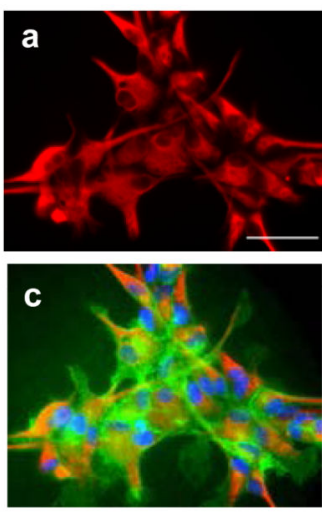

Nestin/EphA2/ DAPI

\section{EphA2}
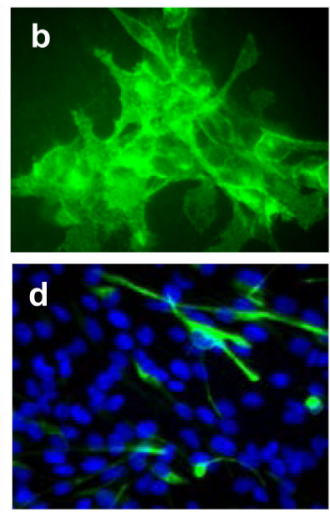

GFAP/DAPI
E
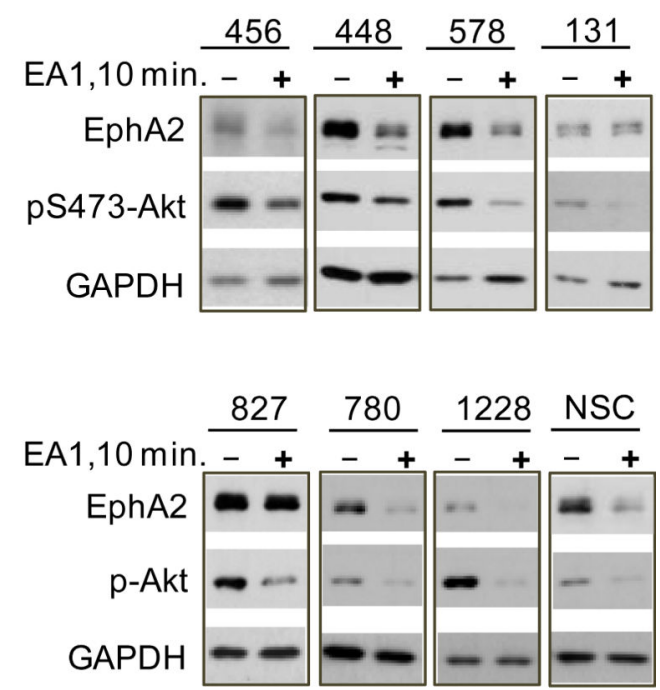

Figure 1.

EphA2 is expressed in glioma stem cells (GSCs) and is phosphorylated on S897. (A) Phase images of GSCs cultured in suspension or on laminin-coated surface. (B) The 827 line of GSCs were cultured on laminin and subjected to immunofluorescence analysis for Nestin (a) and EphA2 (b), which were merged with DAPI in (c). (d) A fraction of GSCs also express GFAP, a differentiation marker. Scale bar, $50 \mu \mathrm{m}$. (C) EphA2 in GSCs was phosphorylated on S897 in the absence of ligand stimulation. Ephrin-A1 treatment led to EphA2 activation, and inhibition of Akt and pS897-EphA2. GSCs cultured on laminin (LM) or in suspension (Sus.) were stimulated with ephrin-A1-Fc and lysed. Whole cell lysates were subjected to immunoblot with the indicated antibodies. (D) Quantitative densitometry analysis shows significant Akt inhibition by the ligand-activated EphA2. Data from 4 independent 
experiments were analyzed. (E) EphA2 receptor is expressed in NSCs and 7 independent preparations of GSCs, and mediates Akt inhibition when stimulated with ephrin-A1 ligand. 
A

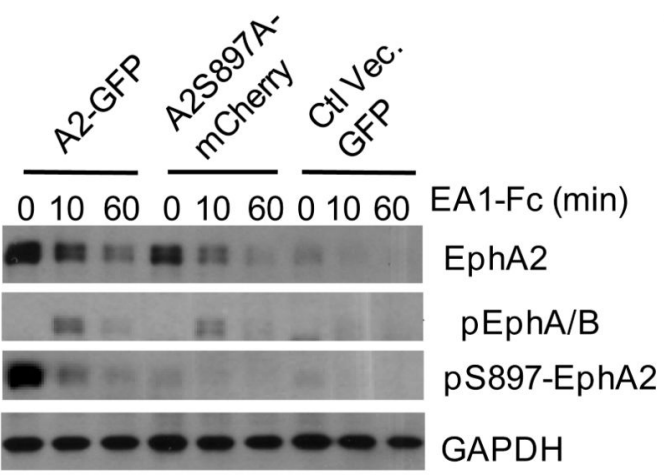

C

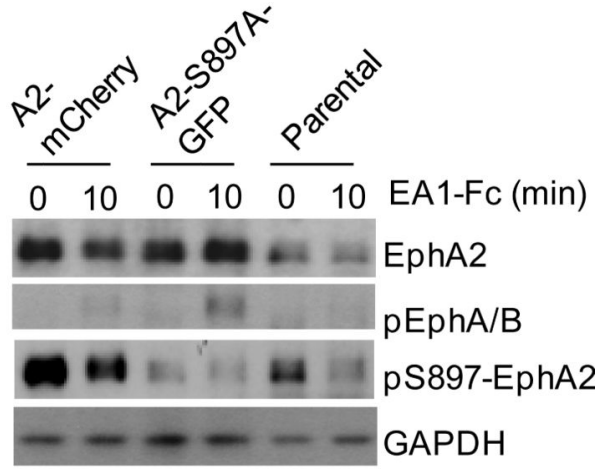

B

Invasion along

Corpus callosum
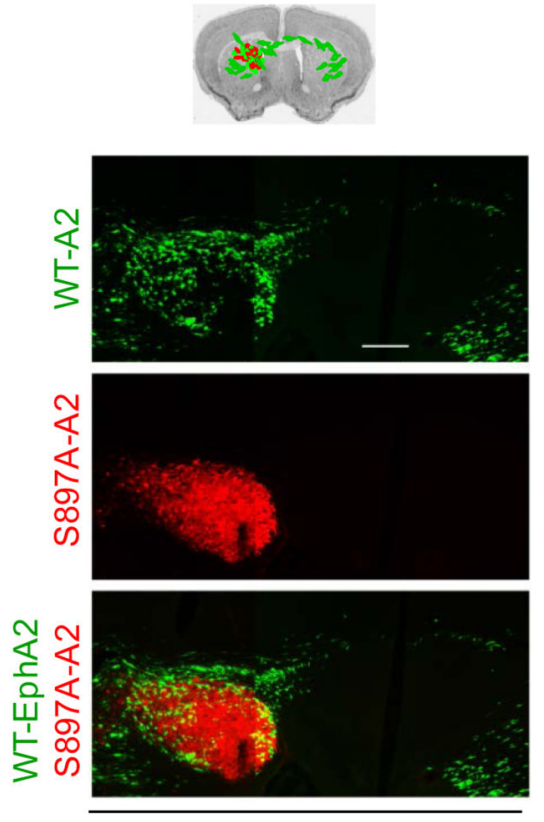

827

D

$\begin{array}{cc}\text { Local } & \text { Distal Subpial } \\ \text { invasion } & \text { invasion }\end{array}$
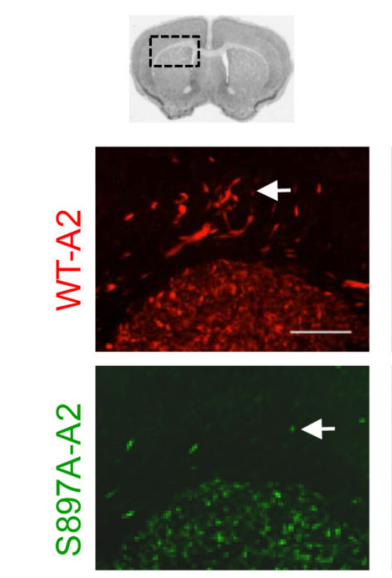

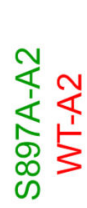
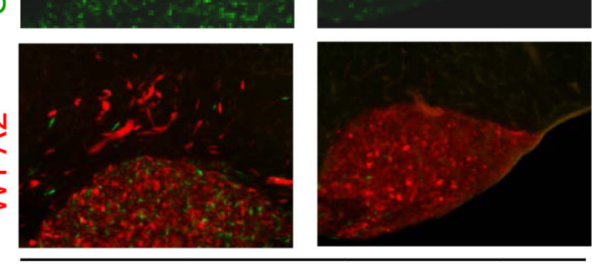

D456MG

Figure 2.

Disruption of Akt-EphA2 signaling axis inhibits the infiltrative invasion of GSCs in vivo. A)

Characterization of 827 GSCs expressing WT- or S897A-EphA2. 827 cells were

sequentially infected first with WT- or S897A-EphA2 retroviral vectors and then with lentiviral vectors expressing eGFP or mCherry. Empty pBabe vector-infected cells tagged with GFP were used as controls. The cells were stimulated with ephrin-A1-Fc and blotted with the indicated antibodies. B) Direct comparison of intracranial invasiveness of WT- vs. S897A-EphA2-expressing 827 cells after co-injection at 1:1 ratio. A representative section shows reduced invasion by S897A-EphA2-mCherry cells (red) compared WT-EphA2-eGFP cells (green). C) Characterization of D456MG GSCs expressing WT- or S897A-EphA2 that were generated following similar strategies as in (A). D) Direct comparison of intracranial invasiveness of WT- vs. S897A-EphA2-expressing D456MG cells after co-injection at 1:1 ratio. S897A mutation significantly reduced invasion both locally (left panels) and distally 
(right panels). Scale bar, $200 \mu \mathrm{m}$. The experiments were performed three times using 6 animals each group. Data from representative experiments are shown. 
A

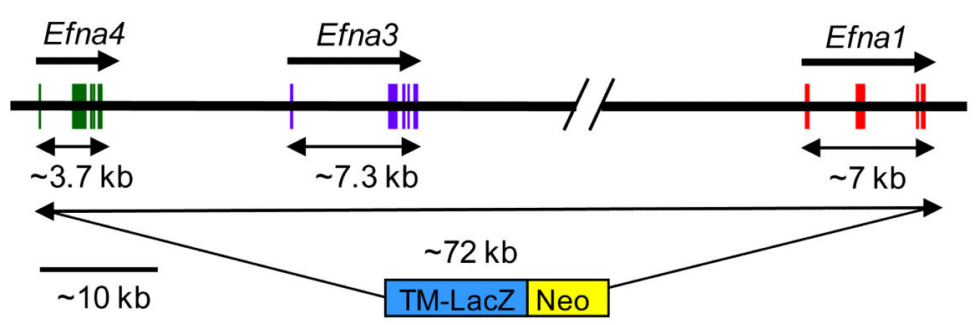

C

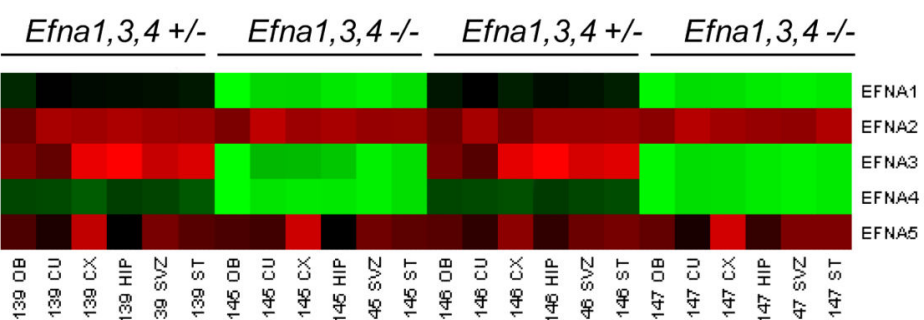

D
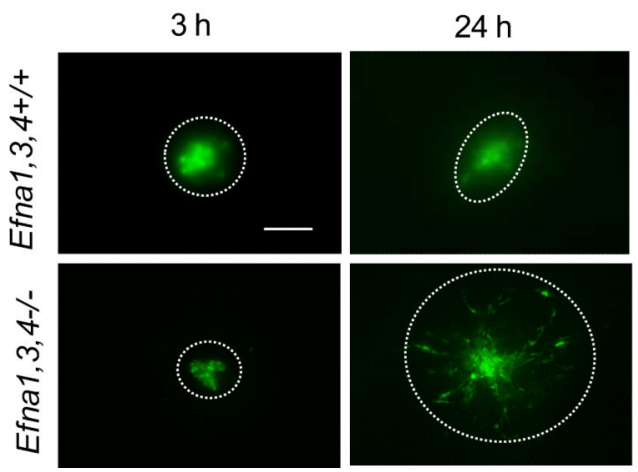

B
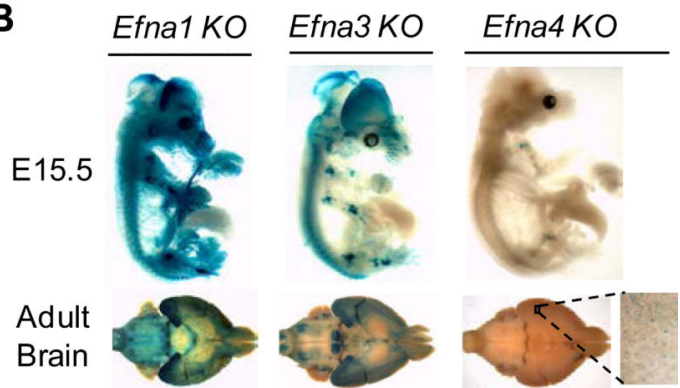

E

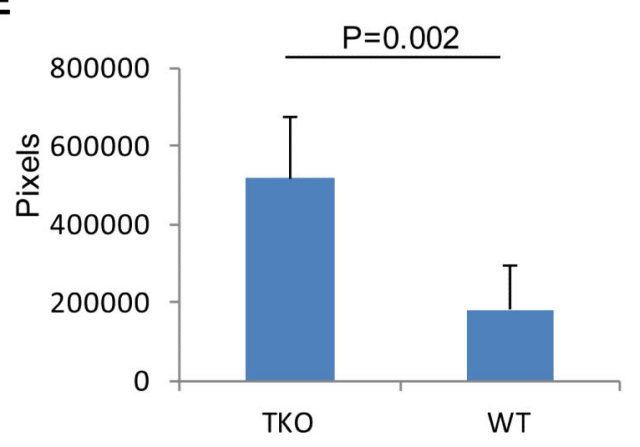

Figure 3.

Establishment of Efnal/Efna3/Efna4 triple knockout (TKO) mice and accelerated ex vivo invasion of GSCs through the mutant brain slices. (A) Schematic illustration depicting the strategies for generating Efnal/Efna3/Efna4 knockout mouse. Note that the 3 Efna genes are localized to a short region that is deleted to generate TKO mice. (B) X-gal staining of E15.5 or adult mouse brains from individual Efna knockout mice. (C) RT-PCR analysis of Efnal-5 gene expression in different regions of the heterozygous or homozygous Efnal,3,4 knockout mice. OB, olfactory bulb; CU, cerebellum; CX, cortex; SVZ, subventricular zone; ST, striatum. (D) Brain slices were prepared from neonatal littermates either wild type or homozygous knockout for Efnal/Efna3/Efna4. The 827 GSCs were tagged with eGFP and implanted in the cortical region near the corpus callosum. Invasion of GSCs was monitored under epifluorescent microscope. Images were collected at the indicated times. Scale bar, $300 \mu \mathrm{m}$. (E) Quantitative analyses of ex vivo invasion assays shown in (D). Total areas covered by the invading GSCs at 3 and 24 hours were measured. The net increases in areas from 3 to 24 hours were compared between the WT and TKO brain slices (n=7) using student $\mathrm{t}$ test. 
A

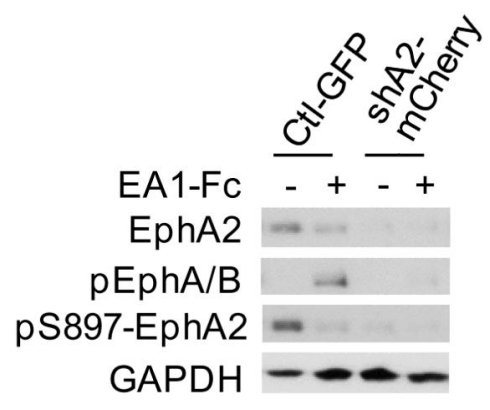

B
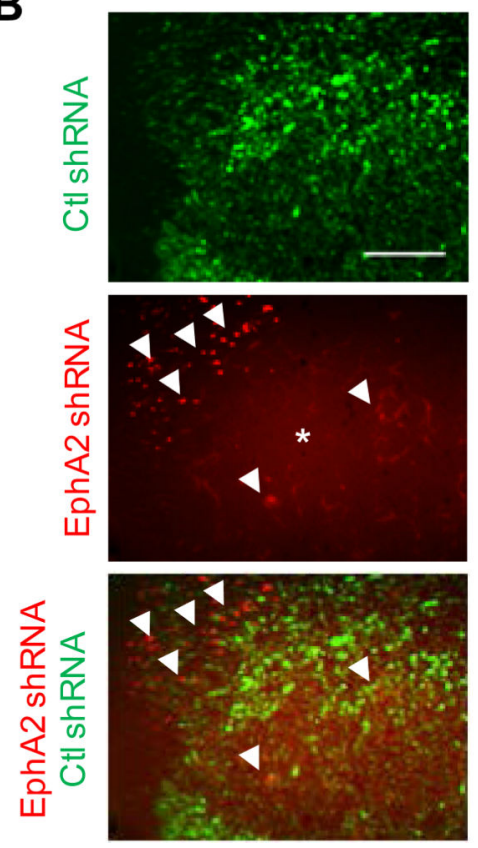

C

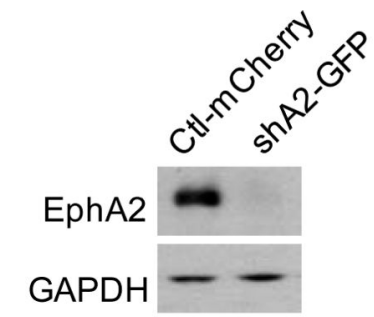

D
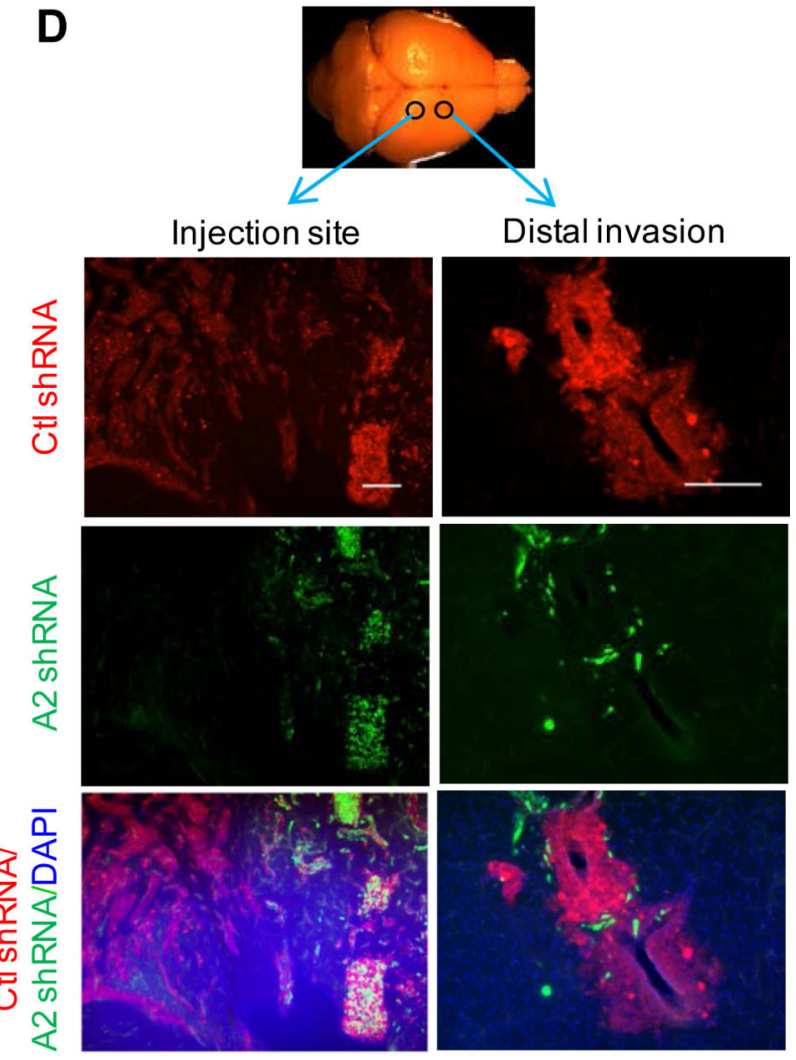

Figure 4.

Knocking down EphA2 expression in 827 cells inhibits in vivo tumor development and reduces expression of stem markers. (A) 827 cells expressing control vector or EphA2 shRNA were tagged with eGFP or mCherry by lentiviral infection, and analyzed by immunoblot with the indicated antibodies. (B) Co-injection of the two cell types are described as in Fig. 2. A representative brain section shows a significantly reduced tumor development by EphA2 shRNA-expressing 827 cells (Arrow heads point to the mCherrytagged tumor cells; star indicates the background fluorescence signal common in mouse brain.) compared with control cells. (C) Silencing EphA2 expression in D456MG cells inhibit the invasion in vivo. D456MG cells were infected with lentivirus expressing control or EphA2 shRNA and then tagged with mCherry or eGFP, respectively. Immunoblot verified the knockdown. (D) Equal number of the two types of cells were mixed and injected as in Fig. 2A. Mice were sacrificed one month later and consecutive sections were prepared from each brain. Scale bars, $200 \mu \mathrm{m}$. The experiments were performed three times using 6 animals each group. Data from representative experiments are shown. 
A

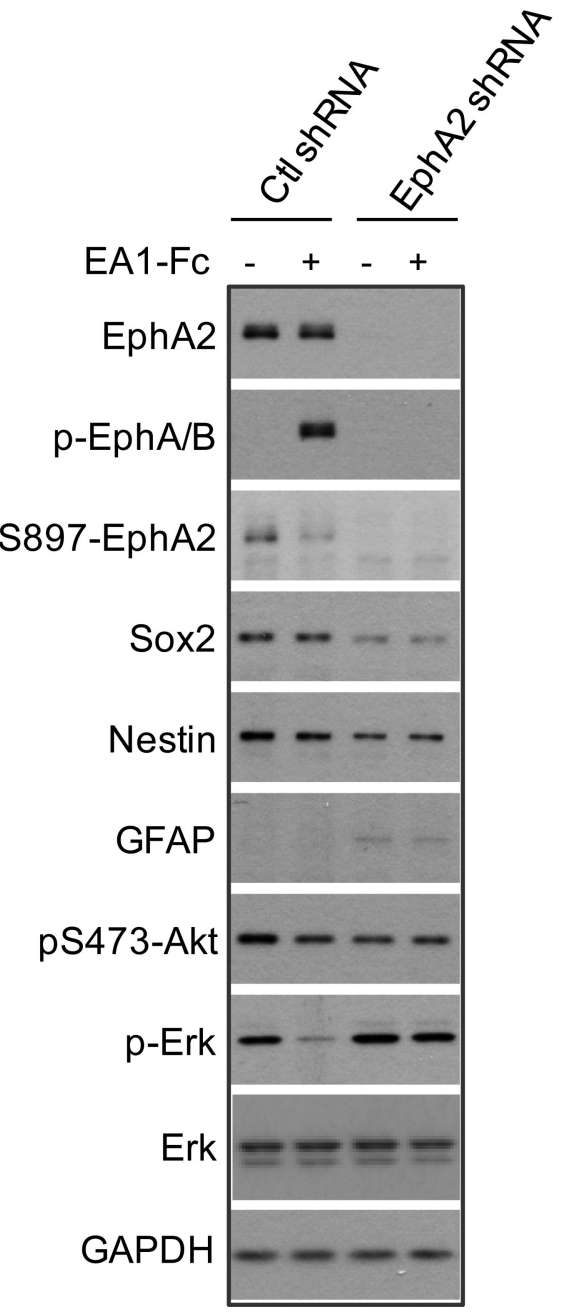

B

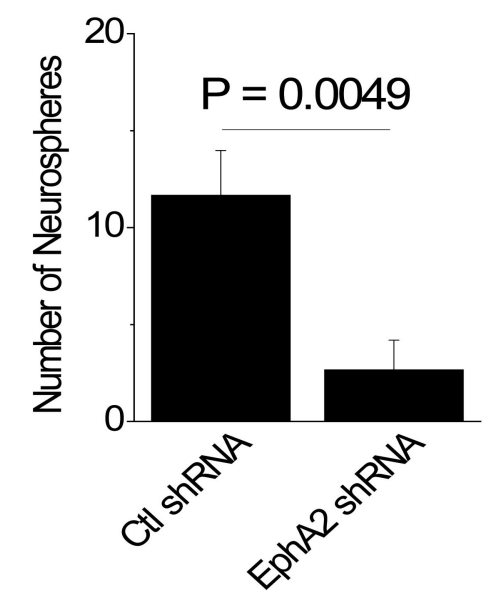

C

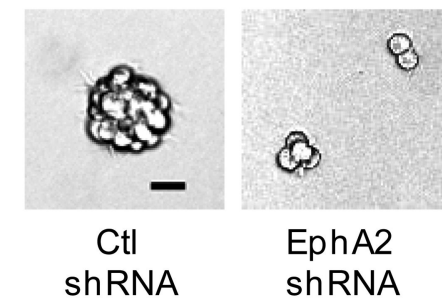

Figure 5.

(A) Knocking down EphA2 in GSCs significantly reduced the expression of stem cell markers including Sox 2 and nestin, and induced the expression of differentiation marker GFAP. 827 cells expressing control vector or EphA2 shRNA were stimulated with ephrinA1-Fc, lysed, and blotted with the indicated antibodies. (B-C) EphA2 is required for the self-renewal of GSCs. One hundred cells were seeded in triplicates in the 6-well ultra-low adhesive plates. The number of neurospheres was counted $(\mathbf{B})$ and representative images $(\mathbf{C})$ were taken 4 days after plating. Scale bar, $25 \mu \mathrm{m}$. 
A

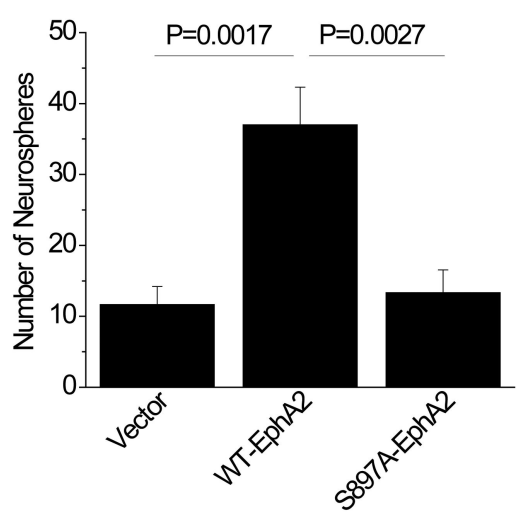

E

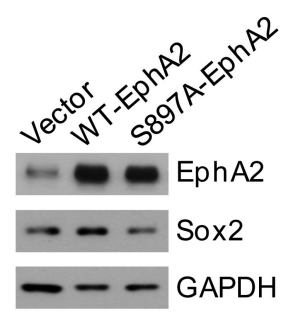

B

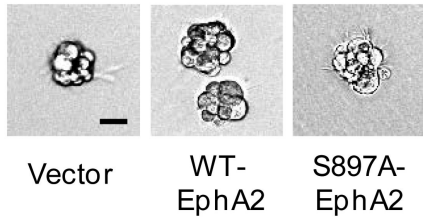

C

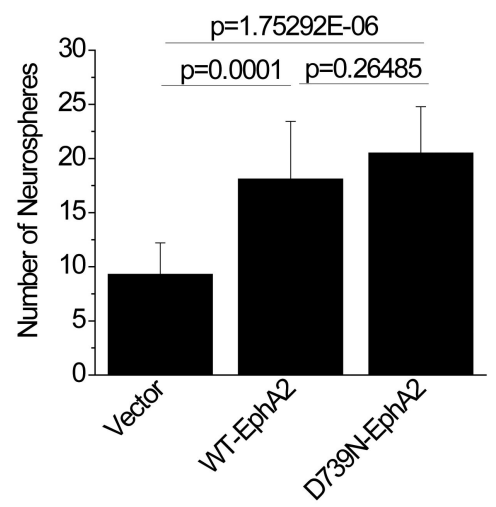

\section{G}

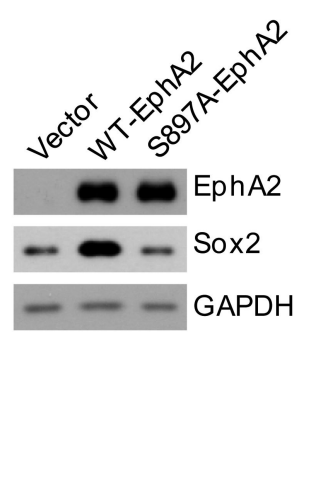

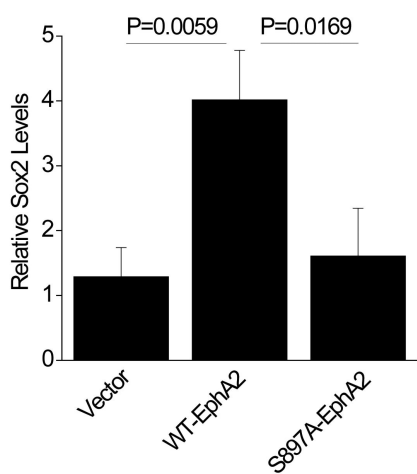

1228

Figure 6.

EphA2 promotes GSC self-renewal and regulates Sox2 expression through crosstalk with Akt. (A-B) Promotion of GSCs renewal requires S897 phosphorylation. One hundred 827 GSCs infected with the indicated retrovirus were seeded in triplicates in the 6-well ultra-low adhesive plates. The number of neurospheres was counted (A) and representative images (B) were taken 4 days after plating. Scale bar, $25 \mu \mathrm{m}$. (C) Both WT- and kinase dead (D739N) EphA2 are capable of promoting neurosphere formation in 827 cells. (D-G) Overexpression of WT- but not S897A-EphA2 increases Sox expression levels in 827 (D,E) and 1228 cells $(F, G)$. Whole cell lysates were prepared from cells cultured on laminin or in suspension and analyzed by immunoblot with the indicated antibodies (D, F). Relative Sox2 levels were determined by normalizing the signal intensity to that of GAPDH. Values represent mean value \pm s.d. from three independent experiments $(E, G)$. 